\title{
Ensino Remoto Emergencial de Disciplinas de Pós-graduação e o Impacto da Pandemia do COVID-19: Um Estudo de Caso
}

\author{
Gabriel Coutinho Natucci - UNICAMP - gabrielnatucci@gmail.com - 0000-0003-4098-0966 \\ Marcos Augusto Francisco Borges - UNICAMP - marcosborges@ft.unicamp.br - 0000-0003-3580-5178
}

\begin{abstract}
Resumo
A pandemia do COVID-19 impactou significativamente escolas, universidades e alunos por todo o globo. Uma estratégia utilizada para mitigar esse impacto é a condução de atividades do tipo ensino a distância; entretanto, essa modalidade apresenta dificuldades naturais em engajamento e motivação dos alunos. Para mitigar esse problema, estratégias mais ativas de aprendizado podem ser utilizadas, como o aprendizado baseado em projetos e teorias construtivistas/construcionistas. Neste contexto, esse artigo busca avaliar o impacto do aprendizado em 35 alunos de pós-graduação de duas disciplinas distintas convertidas para a prática virtual devido a pandemia fazendo uso de conceitos construtivistas $e$ aplicação de projetos. Observou-se uma dificuldade maior nas avaliações e necessidade maior de interações entre aluno-professor, ilustrando possibilidades futuras para cursos em formato híbrido.

Palavras-Chave: aprendizado baseado em projetos, construtivismo, construcionismo, coronavírus, ensino a distância
\end{abstract}

\section{Distance Education of Postgraduate classes and the Impact of COVID-19's Pandemic: A Case Study}

\begin{abstract}
The COVID-19 pandemic caused a significant impact on schools, universities and students across the globe. One approach used to limit this impact is applying online learning classes; however, this approach presents barriers in students' engagement and motivation. To alleviate this problem, common active learning strategies can be implemented, such as project-based learning interventions and constructivism/constructionism approaches. In this context, this article evaluates the learning and cognitive-affectivimpact in two different courses taught to 35 postgraduate students in an online manner, due to the pandemic outbreak. Results found a perceived greater difficulty in performing course evaluations, as well as a greater need to interact with the teacher and other students, showing possibilities beyond the social isolation for blended and hybrid courses.
\end{abstract}

Keywords: project-based learning, constructivism, constructionism, coronavirus, online learning

\section{Introdução}

O surgimento da pandemia de coronavírus (COVID-19) impactou e marcou na história o momento em que o mundo aplicou um forte isolamento social, com fechamentos de instituições fundamentais à sociedade, como instituições de ensino, indústrias e empresas do terceiro setor (incluindo comércio e serviços em geral). Sua disseminação global causou o fechamento de escolas em 107 países até março de 2020 (Viner et al., 2020). Embora seja difícil medir o impacto dessas medidas de fechamento de escolas na contenção da pandemia, é possível estimar como essa medida afeta a escolaridade e instrução dos milhões de alunos afetados; a pandemia pode resultar na perda de até 0.6 anos de escolaridade, levando em conta a qualidade de ensino (Azevedo et al., 2020).

Uma solução para o potencial atraso na educação é a utilização de métodos de ensino a distância (EaD), que não dependam de atividades presenciais, mantendo a política de isolamento social imposta pela pandemia e diminuindo o impacto que a V. $19 \mathrm{~N}^{\mathrm{o}} 1$, julho, 2021 RENOTE 
suspensão total das aulas poderia causar. Ainda assim, vários desafios se colocam para a adoção deste método, como dificuldades na obtenção da infraestrutura necessária para a condução de cursos em larga escala, o nível de preparo dos próprios professores, e até mesmo a dificuldade de acesso virtual por populações em países menos desenvolvidos e comunidades mais pobres (Reich; Ruipérez-Valiente, 2019). Esses desafios e o caráter temporário das mudanças na prática didática dão origem a cursos virtuais na modalidade de ensino remoto emergencial, ou ERE (Hodges et al., 2020), que apesar de sua aplicação em contextos diversos (Gomes et al., 2020) ainda apresenta desafios ligados ao investimento necessário e inclusão digital de alunos (Neves et al., 2021). Neste contexto é especialmente importante considerar o impacto cognitivo-emocional que o isolamento social e o estudar em casa pode ocasionar nos alunos; em casos mais graves, a perda de familiares devido ao COVID-19 pode impactar significativamente qualquer tentativa de aprendizado.

O presente trabalho busca analisar as impressões, sentimentos e opiniões de 35 alunos de pós-graduação da UNICAMP, divididos em duas disciplinas distintas que foram impactadas pela pandemia e tiveram sua experiência transportada para a prática EaD. Considerando os problemas de engajamento que cursos virtuais costumam apresentar, as disciplinas tomaram uma prática pedagógica mais ativa e foram ministradas sob a ótica da aprendizagem baseada em projetos (PjBL), sendo ministradas discussões e aulas expositivas em caráter virtual.

Este artigo está organizado da seguinte forma: a Seção 2 apresenta uma revisão teórica dos principais tópicos de ensino/aprendizagem usados nas disciplinas avaliadas; a Seção 3 descreve a metodologia utilizada para o ensino; a Seção 4 apresenta as observações e resultados obtidos a partir da análise e desempenho dos alunos nas disciplinas e a Seção 4 conclui o artigo, sugerindo trabalhos futuros.

\section{FundamentaçãoTeórica}

O contexto da pandemia torna ainda mais importante a discussão pedagógica sobre o formato e fluxo de oferecimento de disciplinas em ambiente digital. Em particular, o ensino virtual deve apoiar-se ainda mais em conceitos pedagógicos que concedem maior autonomia ao aluno, dada a dificuldade do acompanhamento individual neste meio; práticas construtivistas/construcionistas e métodos de avaliação que vão além de testes escritos, como PjBL são fundamentais para manter o engajamento dos alunos neste momento de crise. Os conceitos de construtivismo/construcionismo serão discutidos na Subseção 2.1; a Subseção 2.2 apresenta o conceito de PjBL e a Subseção 2.3 introduz as práticas e conceitos do ensino a distância e emergencial.

\subsection{Construcionismo e Construtiismo}

O construtivismo é uma linha pedagógica proposta pelo psicólogo Jean Piaget, cuja ideia principal é a de que o aprendizado se dá através da construção interna do conhecimento (Piaget, 1977). Essa nova perspectiva traz algumas implicações: o ensino sempre se dá de forma indireta; o conhecimento é uma experiência adquirida pela interação com pessoas, coisas e o mundo externo (Ackermann, 2001).

A teoria sociocultural de Vygotsky (construtivismo social), foca no conhecimento obtido através de interações sociais com a família e a comunidade (Vygotsky,1978). 
Explora-se o conceito de que o que se pode aprender coletivamente têm influência no conhecimento individual do aprendiz (Eun, 2019).

O construcionismo é uma teoria desenvolvida por Seymour Papert baseada no construtivismo; trata-se do uso do computador como uma ferramenta para a construção do conhecimento (Papert, 1986). Ela difere-se principalmente por ser mais situacional e pragmática (Ackermann, 2001) e por advogar o aprendizado pelo fazer.

\subsection{Aprendizado Baseado em Rajetos}

$\mathrm{O}$ aprendizado baseado em projetos, do inglês project-based learning (PjBL) é uma perspectiva de ensino abrangente que envolve o engajamento de estudantes através do processo investigativo, criando um projeto em busca de soluções a problemas não triviais em um contexto real (Bell, 2010). Esse método incorpora elementos do aprendizado baseado em problemas (PBL), como o uso de questões e problemas para contextualizar o aprendizado (Thomas, 2000), num processo ativo de participação, que envolve iniciativa para explorar novas formas de realizar uma atividade (Chu et al., 2017).

Os benefícios do PjBL têm um efeito positivo no desempenho dos alunos quando comparados aos métodos tradicionais (Chen; Yang, 2019). Para disciplinas ligadas às áreas de ciências e tecnologia (STEM), o PjBL pode ser até $86 \%$ mais eficiente que os métodos tradicionais (Balemen; Keskin, 2018).

\subsection{Ensino a Distância e Ensino Remoto Ergencial}

Embora haja pouco consenso sobre a diferença entre o ensino a distância (EaD), e-learning e ensino online (Moore et al., 2010), é possível definiro $\mathrm{EaD}$ de forma abrangente como uma forma de entrega online de conteúdo, educação, informação e treinamento (Sloman, 2001), com algumas de suas atividades podendo ser realizadas no modo offline, como o ato de assistir vídeos, completar tarefas ou praticar as habilidades adquiridas (Chang, 2016).

Devido à abrangência do termo, é possível aplicá-lo de diversas formas no ambiente educacional. Destacam-se: (1) a abordagem do tipo broadcast, na qual o aluno consome conteúdo com nenhuma interação entre os alunos e o professor, com foco concentrado no material instrucional; (2) a abordagem tipo live e "estar junto", na qual o professor está auxiliando o aluno a resolver problemas e discutir tópicos de forma virtual, com interações intensas e simulando as trocas que ocorrem em uma sala de aula física; (3) a abordagem de ensino híbrido (blended learning), combinando atividades em sala de aula física com práticas de $\mathrm{EaD}$ (Valente, 1993); e (4) a abordagem de cursos online massivos (MOOC), na qual o conteúdo é distribuído na forma de broadcast, porém com foco na distribuição ubíqua de conteúdo e ferramentas automatizadas de avaliação.

O ERE difere-se do $\mathrm{EaD}$ por seu caráter temporário de aplicação devido ao isolamento social consequente da pandemia do Covid-19. Seu objetivo não é criar um sistema robusto de aprendizado online, mas sim fornecer ferramentas e conteúdos temporários para a prática de ensino (Hodges et al., 2020); entretanto, as modalidades de EaD podem ser utilizadas em práticas ERE, através da disponibilização de lives e vídeos pré-gravados em plataformas de distribuição (Moreira et al., 2020). Apesar da prática ser difundida no último ano, o ERE ainda apresenta problemas ligados ao uso de tecnologia e engajamento (Alfaro et al., 2020; Gomes et al., 2020). 


\section{Metodologia}

Para analisar o impacto da pandemia no aprendizado em cursos de ensino superior, optou-se pela realização de um estudo de caso, caracterizado por uma descrição abrangente de um fenômeno em particular (Starman, 2013). O fenômeno avaliado foi a realização de duas disciplinas em cursos de pós-graduação ministradas pelo mesmo professor: uma disciplina regular para alunos de mestrado, doutorado e potenciais interessados em adquirir uma formação stricto sensu, com carga de 60h, divididas em 15 aulas semanais com 4 horas-aula de duração, voltada para o uso de tecnologias na educação (disciplina A); uma disciplina componente de um curso de especialização em governança de Tecnologia da Informação (TI), voltada para a gestão de projetos em tecnologia, com carga horária de 32 horas divididas em quatro sábados entre 9 e 18hs com 1 hora de almoço (disciplina B).

Ambas as disciplinas já existiam antes da pandemia, e foram ministradas na modalidade ERE pela primeira vez no primeiro semestre de 2020, devido ao fechamento das instalações físicas da universidade em função das medidas de isolamento social. Ambas utilizaram a modalidade live na qual alunos e professor interagem em tempo real no horário reservado às disciplinas, realizadas com o auxílio da ferramenta Google Meet; a disciplina A foi ministrada às segundas-feiras das $8 \mathrm{hrs}$ às $12 \mathrm{hrs}$ da manhã. Quanto a materiais instrucionais extras e comunicação assíncrona com os estudantes, foi utilizada a plataforma Google Classroom para a disciplina A e a plataforma Moodle para a disciplina B. A divergência das plataforma utilizadas nas turmas é justi ficada pela diferenciação de contexto das disciplinas: enquanto A é uma disciplina independente, voltada a pós-graduandos dos cursos de Mestrado e Doutorado (stricto sensu), a disciplina B é integrante de um programa de pós-graduação lato sensu, devendo portanto aderir aos métodos e ferramentas utilizadas pelas demais disciplinas deste programa.

Considerando a metodologia pedagógica, ambas as disciplinas foram realizadas de forma construcionista/construtivista; em particular, foi feito amplo uso do PjBL. Para a disciplina $\mathrm{A}$, os projetos consistiram em desenvolver um artigo acadêmico, seguido de sua apresentação online, escrever um capítulo de livro, seguida da apresentação de um seminário. Neste caso, todos os projetos propostos tinham como tema uni ficador tópicos em informática na educação. Para a disciplina $\mathrm{B}$, devido ao número menor e concentrado de intervenções, foi proposta a execução de um projeto envolvendo a desenvolvimento e divulgação de material de apoio sobre algum conceito ligado à gestão de projetos ágeis. Similarmente à disciplina A, os alunos organizaram-se em grupos para a execução desse projeto, porém a metodologia de gestão de projetos foi padronizada, sendo que os alunos deveriam obrigatoriamente usar a metodologia Scrum (Sutherland, 2014) para seu gerenciamento. É importante ressaltar que parte das lives em ambas as disciplinas foi reservada para a condução do projeto, mas em sua maioria as atividades de projeto foram conduzidas de forma independente pelos alunos fora dos horários das disciplinas; as lives portanto consistem em grande parte de aulas expositivas e discussões entre os alunos, bem como de apresentações dos projetos propostos.

Para avaliar o desempenho e impressões dos alunos quanto ao ERE em meio à pandemia, optou-se pela condução de uma análise quantitativa e qualitativa, através da 
construção e distribuição de formulários de pesquisa (survey) usando a plataforma Google Forms. Para ambas disciplinas, o questionário foi composto por três partes: perguntas referentes à atuação profissional, gênero e formação do aluno; perguntas referentes às impressões e experiências da disciplina avaliada; e questões referentes à experiência do aprendizado online e adaptação da metodologia de ensino em função da pandemia. As perguntas foram construídas com respostas do tipo múltipla escolha, com algumas perguntas na escala Likert e outras com comentários abertos; esses últimos estavam focados na atuação do professor, em autoavaliação do aluno, em dicas/sugestões para melhorar o oferecimento da disciplina e, finalmente, no impacto do ensino online para o aprendizado. Para a última seção, as perguntas foram direcionadas ao impacto da condução online na disciplina; ao impacto das avaliações devido à mudança; ao sentimento do aluno em relação a alteração devido à pandemia; ao tempo investido pelo aluno na modalidade online comparado a aula física; e, finalmente, se o formato da aula em futuros oferecimentos deveria ser mantido, retornado às aulas presenciais ou reformulado com formas híbridas de ensino. Após a aplicação da pesquisa e conclusão das disciplinas, os resultados foram compilados em estatísticas simples e análises gráficas, ilustradas na seção a seguir.

\section{Resultados e Discussões}

A aplicação do formulário de pesquisa resultou na coleta de dados de 15 alunos para a disciplina A, e 20 alunos para a disciplina B, após encerramento formal das aulas online.

Com relação a disciplina $\mathrm{A}, 40 \%$ dos respondentes são do gênero feminino, enquanto na disciplina B $30 \%$ representam esse gênero. Em termos de faixa etária, em ambas as disciplinas, a maioria dos alunos encontra-se em idade entre 26-35 anos (40\% na disciplina A; $50 \%$ em B) e 36-45 anos (33,34\% em A; 35\% em B). Alguns poucos alunos ainda encontram-se na faixa dos 46-55 anos (20\% em A; $10 \%$ em B) e somente um aluno de cada disciplina possui menos de 25 anos. Em termos de formação acadêmica, 66,67\% dos alunos da disciplina A possuem formação superior em TI, enquanto os restantes possuem formação superior em áreas diversas (licenciatura, exatas). $46,67 \%$ dos alunos possuem título de pós-graduação stricto sensu, e $40 \%$ possuem título de pós-graduação lato sensu. Já na disciplina B, $80 \%$ possui ensino superior em TI. Os demais (20\%) possuem formação de nível superior em exatas ou em outra área de conhecimento. Do total, 35\% dos alunos possui título de pós-graduação lato sensu.

Considerando a atuação profissional, na disciplina A, 33,34\% dos alunos atua em TI, 46,67\% atua com docência e os 13,33\% restantes dedicam-se à formação acadêmica. $\mathrm{Na}$ disciplina $\mathrm{B}, 65 \%$ dos alunos atuam profissionalmente em TI, 25\% atua em empresas de base tecnológica em outras áreas, e os $10 \%$ restantes atuam em gestão e empreendedorismo.

\subsection{Análise da Disciplina}

A satisfação quanto ao conteúdo ministrado e as avaliações (projetos) propostas foi alta entre os alunos; todos indicaram que tópicos importantes relativos à tecnologias educacionais foram abordados, bem como as avaliações foram condizentes em sala de aula. Isso mostra que a transição para o ERE não afetou o material didático da disciplina, além de indicar a adequação de práticas construtivistas em ambientes 
digitais. Ao analisar o conteúdo proposto, $73,3 \%$ dos alunos se sentiram desafiados e motivados com o tema e trabalhos, e $46,7 \%$ ficaram curiosos com os conteúdos e potencial de aprendizado; $60 \%$ dos alunos julgam a disciplina essencial para sua atuação profissional, e $40 \%$ indicam que ela é importante neste quesito.

Considerando a autoavaliação dos alunos em termos de participação, dois deles reportaram dificuldades familiares devido à pandemia que impactaram seu esforço e desempenho na disciplina; um deles afirmou que teria uma maior participação se a disciplina fosse ministrada presencialmente. Apesar disso, a dedicação dos estudantes foi alta, com 33,3\% deles indicando uma dedicação de mais de 5 horas de estudo semanais, além das aulas ministradas. Com relação a dedicação, $60 \%$ afirmou não ter sido impactado pela mudança, enquanto os demais dividiram-se igualmente em menor e maior dedicação devido a alteração. Dois alunos que reportaram uma maior dedicação à disciplina online tiveram ainda uma dedicação menor do que aqueles que não reportaram alterações. Isso pode sugerir que os alunos confundiram o impacto da nova modalidade com a aplicação de PjBL, prática que naturalmente exige maior dedicação e tempo do aprendiz.

A maioria dos alunos julgou que as mudanças não impactaram na apresentação do conteúdo (60\%); 13,3\% dos alunos ainda reportaram a possibilidade de trabalhar mais conteúdos em EaD/ERE, enquanto $20 \%$ afirmou que houve menos conteúdos trabalhados; somente um aluno reportou uma perda significativa. Em termos de avaliação, $80 \%$ dos alunos não reportaram diferenças quanto à modalidade presencial; dois alunos indicaram que as avaliações foram mais simples $(13,3 \%)$ e somente um reportou maior dificuldade. Quanto aos sentimentos evocados pela mudança na pandemia, $80 \%$ se mostraram motivados com a alteração e $60 \%$ reportaram-se desafiados frente ao uso de novas tecnologias; entretanto, 26,67\% dos alunos demonstraram sentimentos negativos quanto a mudança (receio/medo, tédio/desânimo). Apenas um aluno cogitou desistir da disciplina devido ao novo formato.

Em termos de novos oferecimentos, $60 \%$ dos alunos indicaram preferir uma abordagem de ensino híbrida, com intervenções presenciais e à distância. Os outros $40 \%$ estão divididos entre uma preferência por aulas totalmente presenciais ou totalmente online. Segundo o levantamento feito com os alunos, os principais obstáculos reportados para a adoção de um curso totalmente à distância são a dificuldade de interagir com integrantes do grupo, professores e demais alunos. Algumas pessoas reportaram dificuldades na comunicação com sua equipe de trabalho na modalidade EaD/ERE, se sentindo desconfortáveis com um método de interação totalmente digital. Essa necessidade de interação presencial pode justificar a preferência por cursos híbridos, nos quais a interação com grupos de trabalho, professores e alunos pode ser desenvolvida presencialmente, enquanto o material didático, aulas expositivas e qualquer outra dinâmica instrucional pode ser realizada à distância.

A análise da disciplina sugere que, mesmo para um público com alto grau de instrução e competente no uso de tecnologias, o ensino presencial ainda é preferível para a condução de discussões em grupo, interações entre alunos e conversas abertas com o professor. Houve ainda um interesse em transformar as aulas expositivas, que neste oferecimento foram ministradas em lives, em conteúdos pré-gravados e acessíveis digitalmente, na modalidade broadcast (40\% dos alunos) e MOOC (33,37\% dos alunos). Mesmo nesses casos, foi reforçada a opinião de que as interações entre os pares envolvidos na disciplina não deveriam ser perdidas. 


\subsection{Análise da Disciplina B}

Diferentemente da disciplina anterior, não houve unanimidade quanto a análise do conteúdo ministrado e avaliações: dois alunos (10\%) afirmaram que o conteúdo proposto foi cumprido apenas parcialmente, enquanto que $25 \%$ dos alunos julgaram o método de avaliação apenas parcialmente coerente. Esse fato aponta que a transferência para umadinâmica virtual causou um impacto negativo nessa disciplina, cuja proposta é inerentemente prática. No entanto, os alunos mostraram-se motivados $(75 \%)$ e desafiados $(55 \%)$ com o conteúdo ministrado.

Considerando a autoavaliação de desempenho, nenhum problema foi reportado devido à pandemia. A dedicação dos estudantes ao longo da semana foi bem distribuíctam 30\% dos alunos dedicando mais de 5 horas semanais de estudo, 25\% dedicando de 1 a 2 horas, $20 \%$ de 3 a 5 horas, $15 \%$ de 2 a 3 horas e o restante gastando até 1 hora. De forma similar à disciplina $\mathrm{A}$, os alunos reportaram um impacto em sua dedicação devido à mudança para ERE: $40 \%$ afirmaram ter se dedicado mais nesta modalidade, enquanto $45 \%$ afirmaram que não houve mudança na dedicação; os restantes acreditam que a modalidade foi mais fácil quando comparada à didática presencial.

A valiando a disponibilização de conteúdo, verificou-se uma diferença significativaem relação à disciplina A. Neste caso, $50 \%$ dos alunos afirmaram que o conteúdo foi levemente impactado pelo formato online, perdendo um pouco sua clareza e potencial de engajamento. Entretanto, 20\% dos alunos afirmaram a capacidade de trabalhar conteúdos adicionais devido à mudança, e 25\% declararam não sentir mudanças significativas. Em termos de avaliações (projetos) do curso, foi demonstrado o mesmo impacto negativo da alteração: $35 \%$ julgaram as avaliações em ambiente virtual mais difíceis devido ao formato. Apesar desta dificuldade, somente um aluno (5\%) cogitou desistir da disciplina devido ao impacto da pandemia. Esse aumento na dificuldade em relação à disciplina A pode ser explicado pela aplicação da metodologia ágil Scrum, exigindo que os alunos tivessem reuniões frequentes e gravassem suas reuniões virtuais. A própria natureza da metodologia exige uma interação e cobrança maior entre integrantes do projeto, o que pode levar a um maior sentimento de pressão dos colegas e contribuir para o sentimendo maior dificuldade percebida. O uso desta metodologia pode indicar uma limitação da aplicação de práticas de $\mathrm{PjBL}$ em um contexto EaD/ERE, indicando que os aprendizes devem ter a liberdade para escolher suas próprias metodologias de trabalho, aumentandøssim a autonomia dos aprendizes, diminuição no sentimento de pressão por pares e reforçando o contexto construtivista da aplicação de projetos.

Curiosamente, houve uma quantidade menor de sentimentos negativos reportados pelos alunos devido à alteração; somente 15\% apontaram desânimo/tédio ao assistir aulas virtuais. Entretanto, houve uma motivação menor por parte dos alunos: $35 \%$ mostraram-se motivados e $65 \%$ desafiados com o uso de novas tecnologias.

Quanto a noyos oferecimentos, as opiniões dos alunos enfatizaram fortemente o elemento presencial como aspecto fundamental da disciplina: $50 \%$ dos alunos gostariam de uma aplicação híbrida, 35\% preferem um oferecimento totalmente presencial e somente $15 \%$ prefere manter o oferecimento EaD/ERE. $60 \%$ dos alunos afirmaram não ter interesse em assistir aulas gravadas da disciplina, enquanto que $25 \%$ dos alunos gostariam de uma aula pré-gravada desde que a metodologia PjBL se mantivesse no curso. Um dos motivos que pode explicar esse desinteresse por aulas virtuais se dá pela adaptação muito rápida do curso em função da pandemia, o que pode ter prejudicado a 
condução dos projetos e interação entre os grupos, que precisaram se adaptar à realidade virtual muito rapidamente. A falta de interação pessoal foi outro ponto levantado pelos estudantes que contribuíram para esse desinteresse. Finalmente, o uso da metodologia ágil por todos os participantes foi levantado como um potencial fator para aumentar a dificuldade do curso, principalmente para aqueles que nunca tiveram contato com a metodologia utilizada. Para mitigar esse problema, alguns alunos propuseram que novos oferecimentos incluíssem práticas lúdicas, como uso de jogos, simulações e gamificação para engajar e motivar alunos com pouco conhecimento no tema.

\subsection{Discussão}

Avaliando as duas disciplinas em conjunto, têm-se que mesmo em um grupo de alunos com alto grau de formação e familiaridade com uso de tecnologias, a transferência para a modalidade virtual apresenta desafios,sendo o maior deles a di ficuldade em interação professor-aluno e entre alunos. Outros problemas reportados foram o aumento da carga horária de dedicação entre os estudantes, fundamentados principalmente na velocidade com a qual a transição física-virtual ocorreu em virtude da pandemia; foram reportados ainda problemas pessoais e familiares devido ao impacto do isolamento social, o que pode têm contribuído para uma desestabilização na rotina dos alunos, e, consequentemente, uma maior dedicação à atividades que envolvessem a entrega de projetos, como as disciplinas avaliadas. Quanto à prática de $\mathrm{PjBL}$, os alunos da disciplina B apresentaram maior dificuldades com o acompanhamento da disciplina e seu processo de avaliação; além disso, foram mais propensos a refutar novos oferecimentos online da disciplina. Essa recusa pode ter se originado devido à escolha da metodologia ágil como método de trabalho padrão em uma proposta $\mathrm{PjBL}$, dado que as práticas ágeis necessitam de comunicação frequente e clara, um conceito que é difícil de obter com encontros virtuais (Berczuk, 2007).

Deve-se ressaltar que apesar dos problemas com o oferecimento remoto, a maior parte dos alunos em ambos os casos prefere futuras disciplinas em modalidade híbrida. Com base nas experiências relatadas e análises da satisfação dos alunos, é possível traçar diretrizes pedagógicas para novos oferecimentos que mitiguem os problemas relatados, usando uma abordagem de ensino híbrida. Contrariando a lógica de uso do $\mathrm{EaD}$ como um simples meio de massificação de aulas pré-gravadas, percebe-se a necessidade de discussões entre alunos e professor, aulas expositivas virtuais usando práticas lúdicas e avaliações rápidas para manter o engajamento do aluno constantemente.

\section{Conclusão}

O presente trabalho apresenta uma análise da transição de duas disciplinas de pós-graduação baseadas em $\mathrm{PjBL}$ de um oferecimento tradicional presencial para a modalidade de ensino remoto emergencial (ERE). Neste contexto, 15 alunos de uma disciplina de pós-graduação stricto sensu sobre informática na educação e 20 alunos de uma disciplina de pós-graduação lato sensu sobre gestão de projetos foram entrevistados em formato survey para avaliar o impacto da pandemia e da mudança de modalidade no aprendizado, nos sentimentos evocados e na dedicação aos estudos. O impacto do isolamento social foi mínimo em termos de sentimentos evocados na disciplina, com 
poucos alunos reportando desânimo ou outro sentimento negativo ligado à pandemia que possa ter afetado seu desempenho acadêmico.

A partir dos dados, foi possível observar a necessidade de uma interação maior entre professor-aluno e entre alunos no ambiente online, particularmente em contextos de $\mathrm{PjBL}$ que exigem comunicação frequente, especialmente durante $\mathrm{o}$ uso de metodologias ágeis. Os alunos também reportaram maior di ficuldade na execução das avaliações da disciplina, Além disso, mesmo durante a pandemia, houve relutância dos entrevistados em perceber aulas expositivas pré-gravadas como uma alternativa válida ao oferecimento físico; houve uma indicação de preferência para oferecimentos em modalidade híbrida após o levantamento das medidas de isolamento, com alunos interagindo em projetos e em discussões com o professor presencialmente, enquanto aulas expositivas fossem ministradas virtualmente em estilo live.

Embora os resultados apontem para a criação de diretivas em cursos virtuais que mantenham o engajamento e motivação dos alunos, o estudo foi conduzido em uma única universidade, sendo necessários novos estudos para veri ficar a validade das conclusões obtidas. Futuros trabalhos podem buscar essa generalização dos resultados, traçando diretrizes para cursos híbridos e conteúdos virtuais que mantenham o engajamento dos alunos, sem perder a capacidade de comunicação e troca de experiências.

\section{Agradecimentos}

O presente trabalho foi realizado com apoio da Coordenação de Aperfeiçoamento de Pessoal de Nível Superior - Brasil (CAPES) - Código de Financiamento 001.

\section{Referências}

ACKERMANN, E. Piaget's Constructivism, Papert's Constructionism: What's the difference? Future of learning group publication,v. 5, n. 3, p. 11, 2001.

ALFARO, L. DA T.; CLESAR, C. T. DE S.; GIRAFFA, L. M. M. Os desafios e as possibilidades do ensino remoto na Educação Básica: um estudo de caso com professores de anos iniciais do município de Alegrete/RS. Dialogia, v. 0, n. 36, p. 7-21, 22 dez. 2020.

AZEVEDO, J. P.; HASAN, A.; GOLDEMBERG, D.; IQBAL, S. A.; GEVEN, K. Simulating the Potential Impacts of COVID-19 School Closures on Schooling and Learning Outcomes: A Set of Global Estimates. [s.1.] The World Bank, 2020. BALEMEN, N.; KESKIN, M. Ö. The effectiveness of project-based learning on science education: a meta-analysis search. p. 18, 2018.

BELL, S. Project-Based Learning for the 21st Century: Skills for the Future. The Clearing House: A Journal of Educational Strategies, Issues and Ideas, v. 83, n. 2, p. 39-43, 29 jan. 2010.

BERCZUK, S. Back to Basics: The Role of Agile Principles in Success with an Distributed Scrum Team. AGILE 2007 (AGILE 2007). Anais... In: AGILE 2007 (AGILE 2007). Washington, DC, USA: IEEE, ago. 2007Disponível em: $<$ http://ieeexplore.ieee.org/document/4293626/>. Acesso em: 3 ago. 2020

CHANG, V. Review and discussion: E-learning for academia and industry. 
International Journal of Information Management, v. 36, n. 3, p. 476-485, 1 jun.

2016.

CHEN, C.-H.; YANG, Y.-C. Revisiting the effects of project-based learning on students' academic achievement: A meta-analysis investigating moderators. Educational

Research Review, v. 26, p. 71-81, 1 fev. 2019.

CHU, S. K. W.; ZHANG, Y.; CHEN, K.; CHAN, C. K.; LEE, C. W. Y.; ZOU, E.; LAU, $\mathrm{W}$. The effectiveness of wikis for project-based learning in different disciplines in higher education. The Internet and Higher Education, v. 33, p. 49-60, abr. 2017.

EUN, B. The zone of proximal development as an overarching concept: A framework for synthesizing Vygotsky's theories. Educational Philosophy and Theory, v. 51, n. 1, p. 18-30, 2 jan. 2019.

GOMES, M. A.; SANT'ANNA, E. P. A. DE; MACIEL, H. M. Contexto atual do ensino remoto em tempos de covid-19: um estudo de caso com estudantes do ensino técnico / Current context of remote teaching in times of covid-19: a case study with technical education students. Brazilian Journal of Development,v. 6, n. 10, p. 79175-79192, 19 out. 2020.

HODGES, C.; MOORE, S.; LOCKEE, B.; TRUST, T.; BOND, M. The Difference

Between Emergency Remote Teaching and Online Learning. 27 mar. 2020.

MOORE, J.; DICKSON-DEANE, C.; GALYEN, K.; CHEN, W.Designing for E-learn, Online, and Distance Learning Environments: Are They the Same? 15 jun. 2010 MOREIRA, J. A.; HENRIQUES, S.; BARROS, D. M. V. Transitando de um ensino remoto emergencial para uma educação digital em rede, em tempos de pandemia. Dialogia, p. 351-364, 2020.

NEVES, V. N. S.; VALDEGIL, D. DE A.; SABINO, R. DO N. Ensino remoto emergencial durante a pandemia de COVID-19 no Brasil: estado da arte. Práticas

Educativas, Memórias e Oralidades - Rev. Pemo, v. 3, n. 2, p. e325271-e325271, 30 mar. 2021.

PAPERT, S. Constructionism: A New Opportunity for Elementary Science

Education. [s.1.] Massachusetts Institute of Technology,Media Laboratory,

Epistemology and Learning Group, 1986.

PIAGET, J. The Origin of Intelligence in the Child.[s.1.] Penguin, 1977.

REICH, J.; RUIPÉREZ-VALIENTE, J. A. The MOOC pivot. Science, v. 363, n. 6423, p. 130-131, 11 jan. 2019.

SLOMAN, M. The E-learning Revolution: From Propositions to Reality. [s.1.] CIPD Publishing, 2001.

STARMAN, A. B. The case study as a type of qualitative research. Journal of

Contemporary Educational Studies/Sodobna Pedagogika,v. 64, n. 1, p. 16, 2013.

SUTHERLAND, J. Scrum: A Revolutionary Approach to Building Teams, Beating

Deadlines, and Boosting Productivity. [s.l.] Penguin Random House, 2014.

THOMAS, J. W.A review of research on project-based learning. 2000

VALENTE, J. A. Computadores e conhecimento: repensando a educação. [s.1.]

Universidade Estadual de Campinas, Núcleo de Informática Aplicada à Educação, 1993.

VINER, R. M.; RUSSELL, S. J.; CROKER, H.; PACKER, J.; WARD, J.;

STANSFIELD, C.; MYTTON, O.; BONELL, C.; BOOY, R. School closure and management practices during coronavirus outbreaks including COVID-19: a rapid systematic review.The Lancet Child \& Adolescent Health, v. 4, n. 5, p. 397-404, 1 maio 2020.

VYGOTSKY, L. S. Mind in society: the development of higher psychological processes. Cambridge: Harvard University Press, 1978. 